\title{
Quorum Sensing-enabled Amplification for Molecular Nanonetworks
}

\author{
Sergi Abadal, Ignacio Llatser, Eduard Alarcón, Albert Cabellos-Aparicio \\ NaNoNetworking Center in Catalonia (N3Cat) \\ Universitat Politècnica de Catalunya \\ C/ Jordi Girona 1-3, 08034 Barcelona, Spain \\ \{abadal,llatser,acabello\}@ac.upc.edu and eduard.alarcon@upc.edu
}

\begin{abstract}
Nanotechnology is enabling the development of devices in a scale ranging from a few to hundreds of nanometers. The nanonetworks that result from interconnecting these devices greatly expand the possible applications, by increasing the complexity and range of operation of the system. Molecular communication is regarded as a promising way to realize this interconnection in a bio-compatible and energy efficient manner, enabling its use in biomedical applications. However, the transmission range of molecular signals is strongly limited due to the large and inherent losses of the diffusion process. In this paper, we propose the employment of Quorum Sensing so as to achieve cooperative amplification of a given signal. By means of Quorum Sensing, we aim to synchronize the course of action of a certain number of emitters, which will transmit the same signal. Under the assumption of a linear channel, such signal will be amplified and thus the transmission range will be consequently extended. Finally, we validate our proposal through simulation.

Index Terms-Quorum Sensing; Synchronization; Molecular Communication; Amplification; Nanonetworks; Wireless NanoSensor Networks; Bio-inspired
\end{abstract}

\section{INTRODUCTION}

Nanotechnology enables the development of nanomachines, that is, devices in a scale ranging from one to a few hundreds of nanometers. These nanomachines are not just the downscaled version of classical devices, but the result of taking advantage of the unique properties of nanomaterials at this scale, possibly following a bottom-up approach [1]. For instance, novel nanosensors are able to detect the presence of virus and other harmful agents [2], or to sense chemical compounds in concentrations as low as one molecule [3].

Still, nanomachines are expected to be capable of performing very simple tasks due to their reduced size and energy constraints. Communication between nanodevices greatly enhances and expands the capabilities of single nanodevices. The operational range of nanodevices is extremely limited as it is their size, and that is why networks of nanomachines (also referred to as nanonetworks) allow the application in larger scenarios [1]. Furthermore, nanonetworks can be used to coordinate tasks and realize them in a distributed manner, achieving higher global complexity while maintaining the energy consumption of single entities low.

One example of such nanonetworks are the Wireless NanoSensor Networks (WNSNs) [4], in which nanosensor motes communicate in order to measure phenomena in a precise, autonomous and non-invasive manner. Numerous applications of WNSNs have been proposed in the biomedical, environmental, industrial and military fields [4], being the biomedical applications the ones that are expected to take the most of the unique features of WNSNs. For instance, intrabody networks are envisaged to provide ultra-accurate new health monitoring systems [5] by gathering data about the level of different substances or the presence of certain agents (e.g. cancer biomarkers) and transmitting it wirelessly to the macroscale.

How nanomachines will communicate is still an important research challenge. Traditional wireless electromagnetic communication, by means of graphene-based nano-antennas, has been proposed to address this issue [5], [6], [7]. These techniques are expected to produce electromagnetic radiation in the $\mathrm{THz}$ range [8], offering low propagation delays and high bandwidth. However, the aforementioned biomedical applications generally demand the use of biostable and energy efficient solutions and it remains unclear whether EM-based techniques will meet these requirements. Either way, many research efforts are focused on systems that will enable the use of these techniques.

In contrast, diffusion-based molecular communication has caught the attention of the scientific community as a novel and promising way to achieve short-range communication between devices in the nanoscale [9]. This new communication paradigm mimics the way cells communicate among them, encoding information into molecules that are released until they eventually reach the receiver, that is, the molecules are physically transported by means of diffusion to the receiver. In this diffusion process, molecules move following concentration gradients in a way that can be mathematically modeled by using the Fick's laws of diffusion [10].

As we can see, molecular communication mechanisms are based on completely different principles when compared to EM-based communications and indeed offer a high degree of biocompatibility and energy efficiency [11]. However, molecular communication also poses important challenges that require the development of radically new principles. One of such challenges is the severe attenuation that molecular signals suffer as they propagate through the medium [9]. In this paper, we address the need for novel amplification schemes 
TABLE I

SCALABILITY OF COMMUNICATION METRICS IN WIRELESS ELECTROMAGNETIC AND MOLECULAR CHANNELS [18]

\begin{tabular}{l|c|c} 
Metric & EM channel & Molecular channel \\
\hline Pulse delay & $\Theta(r)$ & $\Theta\left(r^{2}\right)$ \\
Pulse amplitude & $\Theta\left(1 / r^{2}\right)$ & $\Theta\left(1 / r^{3}\right)$ \\
Pulse width & $\Theta(1)$ & $\Theta\left(r^{2}\right)$
\end{tabular}

[ $r$ : transmission distance]

by presenting and simulating a signal amplification technique based on Quorum Sensing [12], [13], [14] for diffusion-based molecular communication.

The remainder of the paper is organized as follows. In Section II, we show that the attenuation introduced by the molecular channel when transmitting pulses of molecules is a limiting factor for molecular communication. Next, we briefly explain the biological phenomenon of Quorum Sensing in Section III, which is the basis for the signal amplification scheme presented in Section IV. Our proposal is validated by means of simulation, yielding some performance results that are presented in Section V. Finally, conclusions and future work are stated in Section VI.

\section{Problem Statement}

In the molecular communication scenario, molecular transmitters will transmit a message encoded in a variable concentration of communication molecules that will propagate towards the receiver by means of diffusion. Actually, experimental results led to the conclusion that cells can adopt modulation schemes similar to the traditional AM or FM techniques [15]. Since the complexity of nanomachines is expected to be very low, researchers are proposing simple modulation techniques, such as concentration-based ON-OFF modulations that encode information into pulses [16], [17]. In this context the receiver interprets low and high molecular concentrations as "absence" or "presence" of a pulse, decoding the information bit accordingly.

However, recent results on the characterization of the physical channel of diffusion-based molecular communication show that encoding the information to be transmitted into pulses of molecules presents significant challenges [18]. Besides the addition of noise from several sources [19], these pulses suffer a great amount of attenuation, delay and distortion as they propagate through the medium (see Table I). In particular, results show that the amplitude of molecular pulses decreases proportionally to the third power of the transmission distance $r, \Theta\left(1 / r^{3}\right)$ [18]. Please note the difference with the scalability in terms of transmission distance of classical EM techniques ${ }^{1}$ : $\Theta\left(1 / r^{2}\right)$. Hence, reaching certain distances using molecular communication schemes might result unfeasible, due to the constrained power budget of single nanomachines.

To exemplify this issue, we performed two simulations in which several pulses were transmitted to two different receivers located 10 and 25 micrometers away from the

\footnotetext{
${ }^{1}$ In this case, only free space electromagnetic radiation was considered.
}

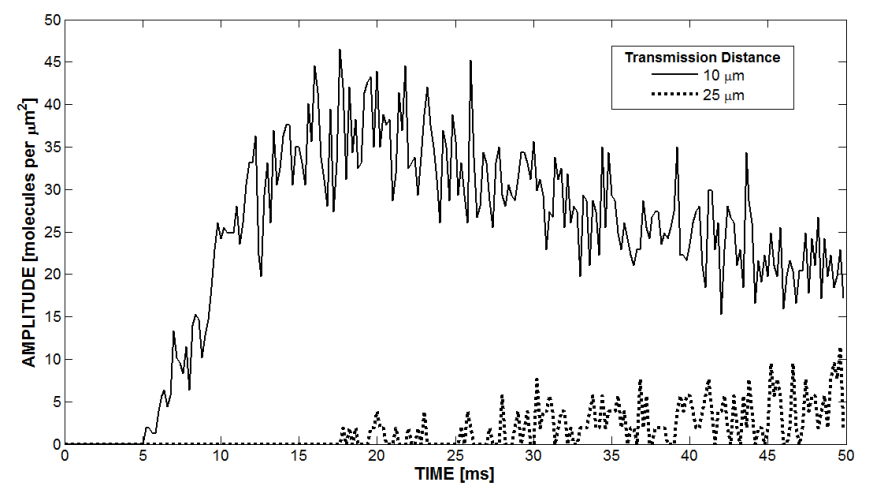

Fig. 1. Reception of a pulse at distances of $10 \mu \mathrm{m}$ (solid line) and $25 \mu \mathrm{m}$ of the transmitter (dotted line).

transmitter. Each pulse was composed of a total of $5 \cdot 10^{5}$ molecules, evenly released by the transmitter during a lapse of time of two milliseconds. It is important to note that these simulations, as well as the other simulations found throughout the paper, were realized using the N3Sim framework. Further details about this simulator can be found in [20].

Figure 1 shows the temporal evolution of the molecular signal received in these two different cases. While the pulse received 10 micrometers away of the transmitter can be easily distinguished, the pulse detected by the distant receiver is highly attenuated and masked by the molecular noise. Consequently and considering a discriminating threshold demodulation scheme, only the first receiver will be able to clearly identify "low" and "high" levels of concentration and thus to decode the message with low error probability.

In conclusion, the attenuation introduced per unit of distance makes the communication only feasible in the very short range. The number of molecules required to successfully cover distances longer than several tens of micrometers can be very large, resulting in a high energy consumption and even exceeding the emission capacity of a single nanomachine. These diffusion losses may even render unfeasible the approach of nodes competing for the channel, traditionally used in current wireless networks. Instead, cooperative schemes where nodes coordinate and jointly transmit the same signal, amplifying it, may allow the implementation of diffusion-based molecular communication. The main challenge then is how to coordinate the action of a group of nanomachines to accomplish the cooperation desired. We propose Quorum Sensing [21] as a way to coordinate the emission of a group of transmitters so that higher distances can be covered while relaxing the power consumption constraints.

\section{QUORUM SENSING}

Quorum Sensing is a biological process that enables the synchronization of a population of bacteria [14]. In order to synchronize with the group, each bacterium releases synchronization molecules at a constant rate. These molecules are called autoinducers since they trigger the release of more particles of the same kind when sensed by other bacteria. 


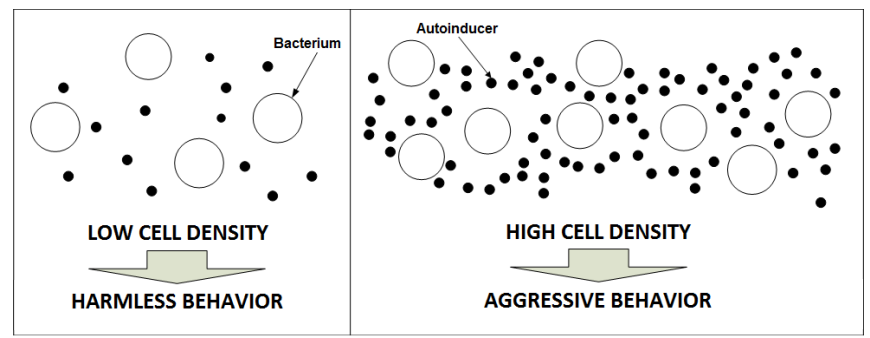

Fig. 2. Quorum Sensing behavior for low and high cell densities.

Hence, the concentration of synchronization molecules in the environment increases proportionally with the bacterial population. This way, bacteria are able to sense their population density by detecting the level of autoinducer concentration in their close environment. When this concentration reaches a critical threshold, the group responds with a synchronized population-wide change of behavior [21].

Quorum Sensing has been described as "the most consequential molecular microbiology story of the last decade" [22] since it is a highly widespread phenomena in the bacterial world. The reason behind this ubiquitous presence is considered to be evolutionary. Quorum Sensing enables the control of bacterial functions that are unproductive when undertaken by a single bacterium but become effective when undertaken by the group; processes that are generally crucial for the species survival. For instance, bacteria species commonly need to activate virulence factors in order to survive or spread. The host's defenses will easily deal with the outbreak of an individual bacterium, whereas the attack of a large group of bacteria will probably result in a successful infection. Many other examples of behaviors controlled by Quorum Sensing can be found in the literature: motility, DNA processing or bioluminescence, amongst others [14], [23].

In previous work, Quorum Sensing principles have been regarded as a way to coordinate the course of action of several nanomachines by means of molecular communication, thus achieving global synchronization in a fully distributed manner [12]. Moreover, bacteria follow a rather simple algorithm with no need of configuration, two characteristics that might be critical if we take into account the intrinsic limitations of nanomachines [13].

Further investigations have reported that some bacteria are able to react to different types of autoinducers sequentially or in parallel, constructively or destructively. This proves that several Quorum Sensing schemes can be combined to implement complex interactions between groups of nanomachines, making use of what has been called "Molecular Division Multiple Access" [24] to significantly expand the potential applications of these systems.

In this paper, we propose to use Quorum Sensing to achieve signal amplification in diffusion-based molecular nanonetworks in a collaborative and distributed manner. The resulting amplification system may help overcome the attenuationrelated limitations that these novel networks show. Further

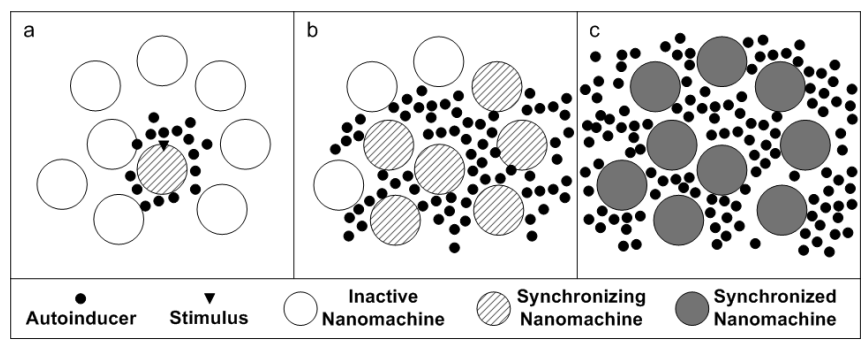

Fig. 3. Detailed evolution of the synchronization process.

details are presented in Section IV, whereas some simulation results can be found in Section V.

\section{Signal Amplification Scheme}

Let us consider that a given number of nodes are deployed randomly over space forming a cluster. There is no a priori synchronization among them and they all have the capacity to emit, detect and distinguish two different types of molecules: autoinducers or synchronization molecules, which will be used to achieve synchronization; and communication molecules, which will be used to encode the message to transmit.

We will also assume that the concentration of molecules is, at all times, sufficiently low so that the probability of collision between particles is negligible. Under this reasonable assumption and in a scenario devoid of external forces, we can consider that the diffusion-based molecular channel is linear. In [16], simulation results have shown that even though molecular diffusion is governed by a nonlinear phenomenon (i.e. Brownian motion), the diffusion process has a linear behavior from a macroscopic perspective. Hence, we can apply the superposition principle, this is, the addition of two received emissions will yield the same signal than the reception of the addition of two emissions.

In this scenario, the proposed amplification scheme has these two following differentiated parts:

1) Synchronization Phase: the principles of Quorum Sensing are applied in this phase, which is represented in Figure 3. When a node has information to transmit to other distant nodes, a given stimulus or command will trigger the release of autoinducers at a constant rate (Fig. 3a). Coherently with the Quorum Sensing autocatalytic mechanism explained in Section III, adjacent nodes detect these synchronization molecules and start emitting molecules of the same type (Fig. 3b). Eventually, the concentration will surpass the preset activation threshold and a transmission cluster of approximately $N$ nodes will activate at a similar time instant (Fig. 3c). The interested reader can find more information about Quorum Sensing-enabled synchronization in [12].

2) Amplification Phase: the cooperative actuation occurs in this part. After the synchronization phase, the original transmitter and its neighbours will jointly and coordinately transmit a given message by using communication molecules. As pointed out above, if we consider that the 


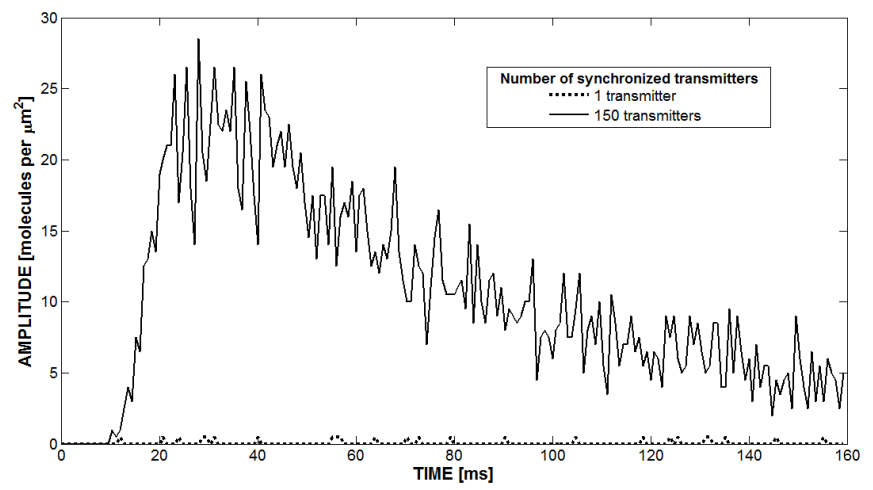

Fig. 4. Reception, at a distance of $50 \mu \mathrm{m}$, of a pulse emitted by one transmitter (dotted line) and of a pulse amplified by a group of 150 transmitters (solid line).

molecular channel is linear [16], the resultant pulse will be received as the sum of the different pulses. Thus, the signal will be effectively amplified.

The specific information encoded into the synchronized signal is left out of the scope of this paper, but it is worth to note that it can be either a pulse, or a (pre)configured sequence of pulses. Furthermore and since hundreds of different autoinducers (synchronization molecules) exist, different autoinducers could trigger different synchronized signals. This way, we can guarantee that all the nodes will transmit the same signal.

\section{Simulation Results}

To exemplify the validity of the proposed scheme, we performed two different simulations in which a 2-millisecond long pulse is transmitted and later picked up by a receiver located at a distance of 50 micrometers from the transmitter area. The pulse is transmitted by a single emitter in the first simulation, whereas a group of 150 synchronized emitters amplifies the original signal in the second case. These 150 emitters are randomly placed in a bounded area, making the distance between emitters practically negligible with respect to the distance to the receiver. In all cases, each emitter has an identical transmission power of 250 molecules per microsecond in either case.

Figure 4 shows the temporal evolution of the particle concentration received in these two different simulations. The receiver was only able to sense residual concentration of particles in the case of individual transmission, while the amplified pulse can be clearly identified in the second case. Eventually and considering a discriminating threshold demodulation scheme, the distant receiver will be able to successfully decode the molecular pulse only if a certain level of signal amplification is achieved at the source.

\section{Signal Amplification}

Figure 5 represents the amplitude of a signal received at a certain distance from the transmission cluster for different numbers of emitters. In other words, it shows the level of

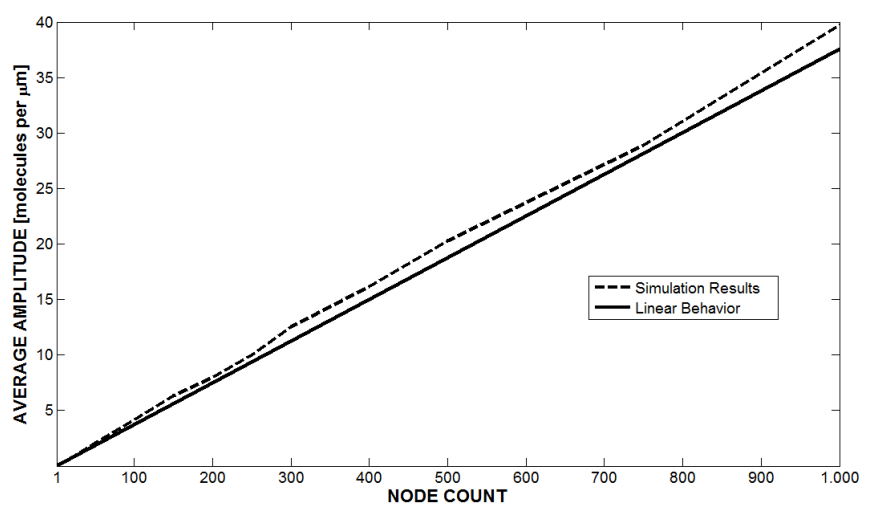

Fig. 5. Average amplitude received at a certain distance as a function of the number of nodes used to amplify the signal.

signal amplification as a function of the size of the transmission cluster. The figure is the result of computing the average number of particles sensed by the receiver during the pulse reception over a given number of repetitions.

As we can see, the received concentration of particles increases almost linearly with the number of emitters of the transmission cluster. Actually, the simulation results are compared with the linear function $Y=q \cdot N$, which is the average concentration received when there was only one emitter (q) multiplied by the node count $(N)$. The slight difference between the two functions remains unclear: it could be either due to inaccuracies in the simulation, but we speculate that a high number of particles could add a certain degree of anomalous diffusion in the diffusion process [25].

\section{Transmission Range Extension}

Assuming that information is encoded into pulses of molecules, the transmission range can be determined by computing the distance at which the received pulses are equal, in terms of amplitude, to the receiver discriminating threshold. Therefore, we can simulate transmissions with different levels of amplification and observe the received amplitude at several distances. The furthest receiver to detect a concentration of molecules above the threshold will be considered to be at the edge of the transmission range. The results are eventually compared with the transmission range obtained for one single emitter.

Figure 6 shows the improvement in transmission range of a cluster of $N$ emitters with respect to the transmission range of a transmission without amplification, as well as its first order derivative. For instance, using a transmission cluster of 100 emitters increases the transmission range almost fivefold, whereas using 1000 emitters means that the transmission range will improve over ten times with respect to the nominal value.

In conclusion, we have seen that the normalized transmission range increases as the number of synchronized emitters grows. However, there is strong evidence of a certain saturation of the amplification when the number of nodes is high. The first order derivative figure represents the differential improvement of adding one more emitter to the transmission 


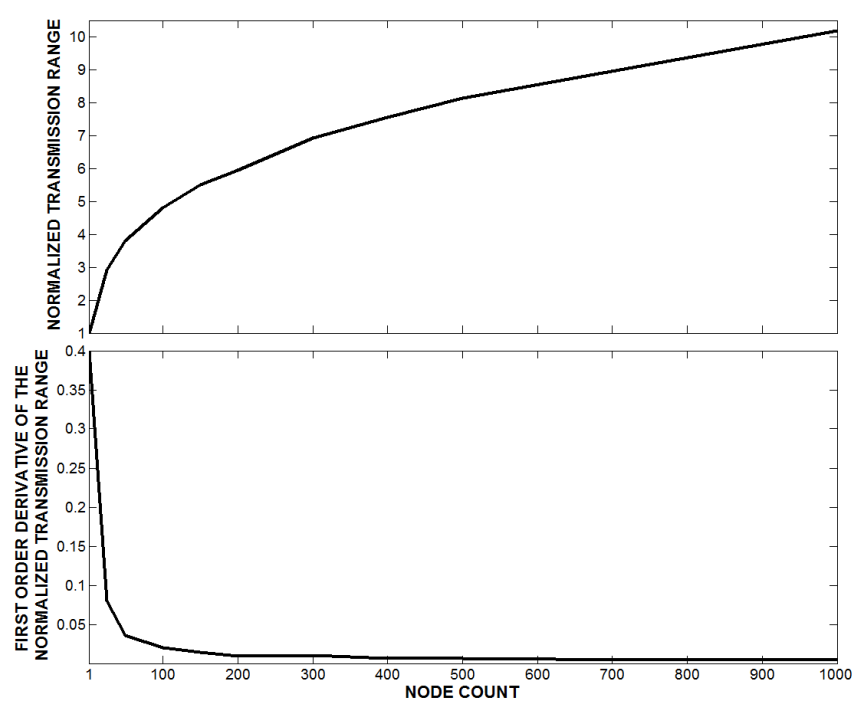

Fig. 6. Normalized transmission range and its first order derivative as a function of the number of nodes used to amplify the signal.

cluster. As we can see, the impact of this inclusion to the overall performance significantly decreases when the number of nodes increases.

\section{CONCLUSIONS AND Future WORK}

The employment of Quorum Sensing in a collaborative scheme, where nodes synchronize with their neighbours and jointly transmit the information, was proposed in this paper. The resulting signal amplification may help overcome the large attenuation present in the diffusion-based communication scenario. We extracted some results after implementing such scheme by means of simulation, such as (1) the linear dependence between the received amplitude and the number of nodes that perform the amplification or (2) the transmission range extension that results from the signal amplification.

The amplification level and, in turn, the final transmission range of the system will depend on the Quorum Sensing process. In future work, we will inspect the synchronization phase that leads to the signal amplification. This way, we expect to provide some insight about how to control the level of amplification or even about the delay introduced by this scheme.

\section{ACKNOWLEDGMENT}

This work has been partially funded by Generalitat de Catalunya (SGR 2009-1140).

\section{REFERENCES}

[1] I. F. Akyildiz, F. Brunetti, and C. Blazquez, "Nanonetworks: A new communication paradigm," Computer Networks, vol. 52, no. 12, pp. 2260-2279, 2008.

[2] Hsiao-Yun Yeh, "Real-time molecular methods to detect infectious viruses," Seminars in cell developmental biology, vol. 20, no. 1, pp. 49-54, 2009.

[3] F. Schedin, A. K. Geim, S. V. Morozov, E. W. Hill, P. Blake, M. I Katsnelson, and K. S. Novoselov, "Detection of individual gas molecules adsorbed on graphene." Nature materials, vol. 6, no. 9, pp. 652-5, Sep. 2007.
[4] I. F. Akyildiz and J. M. Jornet, "Electromagnetic wireless nanosensor networks," Nano Communication Networks, vol. 1, no. 1, pp. 3-19, Mar. 2010.

[5] — "The Internet of nano-things," Wireless Communications, IEEE, vol. 17 , no. 6 , pp. $58-63,2010$.

[6] J. M. Jornet and I. F. Akyildiz, "Graphene-based nano-antennas for electromagnetic nanocommunications in the terahertz band," in Proc. of 4th European Conference on Antennas and Propagation, EUCAP, Ed., Barcelona, 2010.

[7] C. Rutherglen and P. Burke, "Nanoelectromagnetics: circuit and electromagnetic properties of carbon nanotubes." Small (Weinheim an der Bergstrasse, Germany), vol. 5, no. 8, pp. 884-906, Apr. 2009.

[8] J. M. Jornet and I. F. Akyildiz, "Channel Modeling and Capacity Analysis for Electromagnetic Wireless Nanonetworks in the Terahertz Band," IEEE Transactions on Wireless Communications, vol. 10, no. 10, pp. 3211-3221, 2011.

[9] M. Pierobon and I. F. Akyildiz, "Information capacity of diffusion-based molecular communication in nanonetworks," Proc. of IEEE INFOCOM Miniconference, pp. 2-6, 2011.

[10] J. Philibert, "One and a Half Century of Diffusion: Fick, Einstein, Before and Beyond," Diffusion Fundamentals, vol. 4, no. 6, pp. 1-19, 2006.

[11] M. Moore, A. Enomoto, T. Nakano, Y. Okaie, and T. Suda, "Interfacing with nanomachines through molecular communication," IEEE International Conference on Systems Man and Cybernetics, pp. 18-23, 2007.

[12] S. Abadal and I. F. Akyildiz, "Bio-Inspired Synchronization for Nanocommunication Networks," in IEEE Global Telecommunications Conference (GLOBECOM 2011), Houston, TX, USA, 2011, pp. 1-5.

[13] _ "Automata Modeling of Quorum Sensing for Nanocommunication Networks," Nano Communication Networks, vol. 2, no. 1, pp. 74-83, 2011.

[14] J. Henke and B. Bassler, "Bacterial social engagements," Trends in Cell Biology, vol. 14, no. 11, pp. 648-656, 2004

[15] T. Nakano and T. Suda, "Molecular communication through gap junction channels: System design, experiments and modeling," 2nd Bio-Inspired Models of Network, Information and Computing Systems, pp. 139-146, Dec. 2007.

[16] N. Garralda, I. Llatser, A. Cabellos-Aparicio, and M. Pierobon, "Simulation-based Evaluation of the Diffusion-based Physical Channel in Molecular Nanonetworks," Proc. of the 1st IEEE International Workshop on Molecular and Nano Scale Communication (MoNaCom), held in conjunction with IEEE INFOCOM, 2011.

[17] M. U. Mahfuz, D. Makrakis, and H. T. Mouftah, "On the characterization of binary concentration-encoded molecular communication in nanonetworks," Nano Communication Networks, vol. 1, no. 4, pp. 289-300, Dec. 2010.

[18] I. Llatser, E. Alarcón, and M. Pierobon, "Diffusion-based Channel Characterization in Molecular Nanonetworks," in Proc. of the 1st IEEE International Workshop on Molecular and Nano Scale Communication (MoNaCom), held in conjunction with IEEE INFOCOM, 2011

[19] M. Pierobon and I. F. Akyildiz, "Diffusion-based Noise Analysis for Molecular Communication in Nanonetworks," IEEE Transactions on Signal Processing, vol. 59, no. 6, pp. 2532-47, 2011.

[20] I. Llatser, I. Pascual, N. Garralda, A. Cabellos-aparicio, M. Pierobon, E. Alarcón, and J. Solé-Pareta, "Exploring the Physical Channel of Diffusion-based Molecular Communication by Simulation," in IEEE Global Telecommunications Conference (GLOBECOM 2011), Houston, TX, USA, 2011, pp. 1-5.

[21] C. Fuqua, S. Winans, and P. Greenberg, "Quorum Sensing in Bacteria: the LuxR-LuxI Family of Cell Density-Responsive Transcriptional Regulatorst," Journal of Bacteriology, vol. 176, no. 2, pp. 269-275, 1994.

[22] N. Krasnogor, M. Gheorghe, G. Terrazas, S. Diggle, P. Williams, and M. Camara, "An appealing computational mechanism drawn from bacterial quorum sensing," Bulletin of the EATCS, vol. 85, pp. 135-148, 2005.

[23] K. Xavier and B. Bassler, "LuxS quorum sensing: more than just a numbers game," Current opinion in microbiology, vol. 6, no. 2, pp. 191-197, 2003

[24] L. Parcerisa and I. F. Akyildiz, "Molecular Communication Options for Long Range Nanonetworks," Computer Networks, vol. 53, no. 16, pp. 2753-2766, 2009

[25] D. S. Banks and C. Fradin, "Anomalous diffusion of proteins due to molecular crowding." Biophysical journal, vol. 89, no. 5, pp. 2960-71, Dec. 2005. 